\title{
INTEGRAL REPRESENTATIONS FOR PADÉ-TYPE OPERATORS
}

\author{
NICHOLAS J. DARAS
}

Received 2 October 2000 and in revised form 10 October 2001

The main purpose of this paper is to consider an explicit form of the Padé-type operators. To do so, we consider the representation of Padétype approximants to the Fourier series of the harmonic functions in the open disk and of the $L^{p}$-functions on the circle by means of integral formulas, and, then we define the corresponding Padé-type operators. We are also concerned with the properties of these integral operators and, in this connection, we prove some convergence results.

\section{Introduction}

Let $f$ be a function analytic in the open unit disk $D$, with Taylor power series expansion $\sum_{v=0}^{\infty} a_{v} \cdot z^{v}$, and let $\Lambda_{f}$ be the linear functional on the space of complex polynomials defined by $\Lambda_{f}\left(x^{v}\right)=a_{v}(v=0,1,2, \ldots)$. By Cauchy's integral formula and by a density argument, the functional $\Lambda_{f}$ can be extended to the space $A(\bar{D})$ of all functions which are analytic in $D$ and continuous in the open neighborhood of $\bar{D}$ (see [4]). In particular, we have $f(z)=\Lambda_{f}\left((1-x \cdot z)^{-1}\right)$ for any $z \in D$.

Now, let $v_{m+1}(x)$ be an arbitrary polynomial of degree $m+1$, with distinct zeros $\pi_{1}, \pi_{2}, \ldots, \pi_{n}$ of respective multiplicities $\left(m_{1}+1\right),\left(m_{2}+1\right), \ldots$, $\left(m_{n}+1\right)$ and $\left(m_{1}+1\right)+\left(m_{2}+1\right)+\cdots+\left(m_{n}+1\right)=m+1$.

Let $I\left(v_{m+1}\right)$ be the linear operator mapping each $g(x) \in A(\bar{D})$ into its Hermite interpolation polynomial $G_{m+1}$ of degree at most $m$ defined by

$$
g^{(j)}\left(\pi_{i}\right)=G_{m+1}^{(j)}\left(\pi_{i}\right) \quad \text { for } i=1, \ldots, n, j=0,1, \ldots, m_{i} .
$$

If $g(x, z)=(1-x \cdot z)^{-1}$, then $\Lambda_{f}\left(G_{m+1}(x, z)\right)$ is the so-called Padé-type 
approximant to $f(z)$ with generating polynomial $v_{m+1}(x)$. It is a rational function with numerator of degree $m$ and denominator of degree $m+1$, denoted by $(m /(m+1))_{f}(z)$ and such that

$$
f(z)-\left(\frac{m}{m+1}\right)_{f}(z)=O\left(z^{m+1}\right), \quad \text { if }|z|<\min \left\{\frac{1}{\left|\pi_{1}\right|}, \ldots, \frac{1}{\left|\pi_{n}\right|}\right\}
$$

If $v_{m+1}(x)$ is identical to the orthogonal polynomial $q_{m+1}(x)$ with respect to $\Lambda_{f}$, that is, the polynomial satisfying the orthogonality conditions $\Lambda_{f}\left(x^{v} \cdot q_{m+1}(x)\right)=0, v=0,1, \ldots, m$, then the Padé-type approximant $(m /(m+1))_{f}(z)$ becomes identical to the classical Padé approximant $[m /(m+1)]_{f}(z)$ such that

$$
f(z)-\left[\frac{m}{m+1}\right]_{f}(z)=O\left(z^{2 m+2}\right), \quad \text { if }|z|<\min \left\{\frac{1}{\left|\pi_{1}\right|}, \ldots, \frac{1}{\left|\pi_{n}\right|}\right\}
$$

By making use of the notation of duality, we can also write

$$
\begin{aligned}
\left(\frac{m}{m+1}\right)_{f}(z) & =\Lambda_{f}\left(G_{m+1}(x, z)\right) \\
& =\left\langle\Lambda_{f},\left[I\left(v_{m+1}\right)\right](1-x \cdot z)^{-1}\right\rangle \\
& =\left\langle\left[I^{*}\left(v_{m+1}\right)\right]\left(\Lambda_{f}\right)(1-x \cdot z)^{-1}\right\rangle
\end{aligned}
$$

In [3], Brezinski showed that the operator which maps $f$ on $(m /(m+$ 1)) $f$ can be understood as the mapping of $A^{*}(\bar{D})$ into itself which maps $\Lambda_{f}$ into $\left[I^{*}\left(v_{m+1}\right)\right]\left(\Lambda_{f}\right)$. This mapping, which depends on the generating polynomial $v_{m+1}(x)$, is called the Padé-type operator for the space $O(D)$ of all analytic functions on $D$ and it is exactly the operator $I^{*}\left(v_{m+1}\right)$. If $v_{m+1}(x)$ does not depend on $\Lambda_{f}$, then $I^{*}\left(v_{m+1}\right)$ is linear. But for Padé approximants, since $v_{m+1}(x)$ is the orthogonal polynomial $q_{m+1}(x)$ of degree $m+1$ with respect to the functional $\Lambda_{f}$, then $v_{m+1}(x)$ depends on $\Lambda_{f}$ and the linearity property holds only if the first $2 m+2$ moments of both functionals are the same since, then, both orthogonal polynomial of degree $m+1$ will be the same.

The aim of this paper is to consider the explicit form of the Padétype operator by means of integral representations. Section 2 deals with the definition of integral representations of Padé-type approximants to real-valued $L^{2}$ or harmonic functions and, thus, with the expressions of the Padé-type operator for the spaces $L_{\mathbb{R}}^{2}(C)$ of all real-valued $L^{2}$ functions on $C, L_{\mathbb{R},(2 \pi \text {-per })}^{2}[-\pi, \pi]$ of all real-valued $2 \pi$-periodic $L^{2}$ functions on $[-\pi, \pi]$, and $H_{\mathbb{R}}(D)$ of all real-valued harmonic functions on $D$. We 
also give some examples with applications of these integral representations for the Padé-type operator to the convergence problem of a series of Padé-type approximants and to the problem of finding a sufficient condition permitting the interpretation of any $2 \pi$-periodic $L^{p}$ real-valued function on $[-\pi, \pi]$ as a Padé-type approximant. In [7], by introducing the so-called composed Padé-type approximation, we discussed the general situation of complex-valued harmonic or $L^{p}$ functions and we showed that any Padé-type approximant in the ordinary sense to a function $f \in O(D)$ is a special case of this composed procedure. It is therefore natural to reflect that any $I^{*}\left(v_{m+1}\right)$ can also be viewed as a special case of the operator which maps every $f \in O(D)$ on a composed Padé-type approximant to $f$. Such a mapping will be called a composed Padé-type operator for $O(D)$. In Section 3, we define and give the explicit form of the composed Padé-type operators for the spaces $L_{\mathbb{C}}^{2}(C)$ of all complexvalued $L^{2}$ functions on $C, L_{\mathbb{C},(2 \pi \text {-per })}^{2}[-\pi, \pi]$ of all complex-valued $2 \pi$ periodic $L^{2}$ functions on $[-\pi, \pi]$, and $H_{\mathbb{C}}(D)$ of all complex-valued harmonic functions on $D$. Since $O(D) \subset H_{\mathbb{C}}(D)$, we thus obtain the desired explicit form of $I^{*}\left(v_{m+1}\right)$.

\section{Integral representations and Padé-type operators}

In $[5,6]$, we have defined and studied Padé-type approximation to $L^{P} 2 \pi$ periodic real-valued functions and to harmonic functions in $D$. In all cases, the development of our theory was analogous to the classical one about analytic functions.

Really, no situation is quite as pleasant as the $L^{2}$ case. In this section, we look for another way to introduce Padé-type approximants to $L^{2}$ functions and to harmonic functions. Our method is based on integral representation formulas and leads to a number of convergence results.

To begin our discussion, consider any real-valued $L^{2}$ function $u(z)$ defined on the unit circle $C$. Suppose that the Fourier series expansion of $u\left(e^{i t}\right)$ is $\sum_{v=-\infty}^{\infty} \sigma_{v} \cdot e^{i v t}$. Since $u$ is square integrable, the sequence of partial sums $\left\{\sum_{v=-n}^{n} \sigma_{v} \cdot e^{i v t}: n=0,1,2, \ldots\right\}$ converges to $u\left(e^{i t}\right)$ in the $L^{2}$ norm. Let $P(\mathbb{C})$ be the vector space of all complex-valued analytic polynomials with coefficients in $\mathbb{C}$. For every $p(x)=\sum_{v=0}^{m} \beta_{v} \cdot x^{v} \in P(\mathbb{C})$, we denote by $\bar{p}(x)$ the polynomial $\bar{p}(x)=\sum_{v=0}^{m} \bar{\beta}_{v} \cdot x^{v} \in P(\mathbb{C})$. Define the linear functionals $T_{u}: P(\mathbb{C}) \rightarrow \mathbb{C}$ and $S_{u}: P(\mathbb{C}) \rightarrow \mathbb{C}$ associated with $u$ by

$$
T_{u}\left(x^{v}\right):=\sigma_{v}, \quad S_{u}\left(x^{v}\right):=\sigma_{-v} \quad(v=0,1,2, \ldots)
$$

As it is well known, the Poisson integral of $u(z)=u\left(e^{i t}\right)(|z|=1)$ extends to a harmonic real-valued function $u(z)=u\left(r \cdot e^{i t}\right)$ in the unit disk $D$ (where $|z|<1,0 \leq r<1$ ). This harmonic function being the real part of 
54 Integral representations for Padé-type operators

some analytic function in $D$, we immediately see that $\overline{T_{u}\left(x^{v}\right)}=\bar{\sigma}_{v}=\sigma_{-v}=$ $S_{u}\left(x^{v}\right)$ for any $v \geq 0$. More generally, we have the following proposition.

Proposition 2.1. For every $p(x) \in P(\mathbb{C})$ there holds

$$
\overline{S_{u}(p(x))}=T_{u}(\bar{p}(x)), \quad \overline{S_{u}(\bar{p}(x))}=T_{u}(p(x)) .
$$

Proof. Let $p(x)=\sum_{v=0}^{m} \beta_{v} x^{v} \in P(\mathbb{C})$. By linearity, we obtain

$$
\begin{aligned}
S_{u}(p(x))= & S_{u}\left(\sum_{v=0}^{m} \beta_{v} x^{v}\right)=\overline{\sum_{v=0}^{m} \beta_{v} S_{u}\left(x^{v}\right)=\sum_{v=0}^{m} \beta_{v} \overline{T_{u}\left(x^{v}\right)}} \\
= & \overline{\sum_{v=0}^{m} \bar{\beta}_{v} \cdot T_{u}\left(x^{v}\right)}=\overline{T_{u}\left(\sum_{v=0}^{m} \bar{\beta}_{v} \cdot x^{v}\right)}=\overline{T_{u}(\bar{p}(x))} \\
\overline{S_{u}(\bar{p}(x))} & =\overline{S_{u}\left(\sum_{v=0}^{m} \bar{\beta}_{v} x^{v}\right)}=\overline{\sum_{v=0}^{m} \bar{\beta}_{v} S_{u}\left(x^{v}\right)}=\overline{\sum_{v=0}^{m} \bar{\beta}_{v} \overline{T_{u}\left(x^{v}\right)}} \\
& =\sum_{v=0}^{m} \beta_{v} \cdot T_{u}\left(x^{v}\right)=T_{u}\left(\sum_{v=0}^{m} \beta_{v} \cdot x^{v}\right)=T_{u}(p(x)) .
\end{aligned}
$$

Corollary 2.2. For every $p(x) \in P(\mathbb{C})$ there holds

$$
\operatorname{Re}_{u}(\bar{p}(x))=\operatorname{Re} S_{u}(p(x)), \quad \operatorname{Re}_{u}(p(x))=\operatorname{Re} S_{u}(\bar{p}(x)) .
$$

Now, observe that the linear functional $S_{u}$ can be extended continuously on the space $L_{\mathbb{R}}^{2}(C)$ of all real-valued square integrable functions on the unit circle $C$. In fact, if $p(x)=\sum_{v=0}^{m} \beta_{v} x^{v} \in P(\mathbb{C})$ then, by Hölder's inequality, we get

$$
\begin{aligned}
\left|S_{u}(p(x))\right|^{2} & =\left|\sum_{v=0}^{m} \beta_{v} \sigma_{-v}\right|^{2} \\
& =\left|\sum_{v=0}^{m} \bar{\beta}_{v} \sigma_{v}\right|^{2} \\
& =\left|\frac{1}{2 \pi} \cdot \int_{-\pi}^{\pi} u\left(e^{i t}\right) \cdot\left(\sum_{v=0}^{m} \bar{\beta}_{v} \cdot e^{-i v t}\right) d t\right|^{2} \\
& =\left|\frac{1}{2 \pi} \cdot \int_{-\pi}^{\pi} u\left(e^{i t}\right) \cdot \overline{p\left(e^{i t}\right)} d t\right|^{2} \\
& \leq c_{u} \cdot\|p(x)\|_{2}^{2},
\end{aligned}
$$


for some positive constant $c_{u}$ depending only on $u$, and hence, by the Hahn-Banach theorem, there is a continuous linear extension of $S_{u}$ on $L_{\mathbb{R}}^{2}(C)$. It follows, from the Riesz representation theorem, that there exists a unique $F_{u} \in L_{\mathbb{R}}^{2}(C)$ such that

$$
S_{u}(g)=\int_{C} g(\zeta) \cdot \overline{F_{u}(\zeta)} d \zeta=i \cdot \int_{-\pi}^{\pi} g\left(e^{i \theta}\right) \cdot \overline{F_{u}\left(e^{i \theta}\right)} \cdot e^{i \theta} d \theta
$$

for all $g \in L_{\mathbb{R}}^{2}(C)$. In particular, if $g(\zeta)=\zeta^{v}$ then

$$
S_{u}\left(\zeta^{v}\right)=\int_{C} \zeta^{v} \overline{F_{u}(\zeta)} d \zeta=i \cdot \int_{-\pi}^{\pi} e^{i v \theta} \cdot \overline{F_{u}\left(e^{i \theta}\right)} \cdot e^{i \theta} d \theta
$$

But

$$
S_{u}\left(\zeta^{v}\right)=\sigma_{-v}=\frac{1}{2 \pi} \cdot \int_{-\pi}^{\pi} u\left(e^{i \theta}\right) \cdot e^{i v \theta} d \theta
$$

and therefore

$$
\overline{F_{u}\left(e^{i \theta}\right)}=-i \cdot u\left(e^{i \theta}\right) \cdot e^{-i \theta},
$$

which implies that

$$
S_{u}(g)=\int_{-\pi}^{\pi} g\left(e^{i \theta}\right) \cdot u\left(e^{i \theta}\right) d \theta
$$

for all $g \in L_{\mathbb{R}}^{2}(C)$. In view of Corollary 2.2, we have thus obtained the following theorem.

THEOREM 2.3. Let $M=\left(\pi_{m, k}\right)_{m \geq 0,0 \leq k \leq m}$ be an infinite triangular interpolation matrix with complex entries and, for any $m \geq 0$, let $G_{m}(x, z)$ be the unique polynomial of degree at most $m$ which interpolates the function $(1-x \cdot z)^{-1}$ at $x=\pi_{m, 0}, \pi_{m, 1}, \pi_{m, 2}, \ldots, \pi_{m, m}$ (where $z$ is fixed and $\left|\pi_{m, k}\right|<1$ ).

(a) For any real-valued function $u \in L_{\mathbb{R}}^{2}(C)$, the Padé-type approximant $\operatorname{Re}(m /(m+1))_{u}(z)$ to $u(z)$ has the following integral representation:

$$
\operatorname{Re}\left(\frac{m}{m+1}\right)_{u}(z)=\frac{1}{2 \pi} \cdot \int_{C} u(\zeta) \operatorname{Re}\left\{\frac{4 \pi \bar{G}_{m}(\zeta, z)-1}{i \zeta}\right\} d \zeta \quad(|z|=1)
$$


56 Integral representations for Padé-type operators

or equivalently

$$
\begin{aligned}
\operatorname{Re}\left(\frac{m}{m+1}\right)_{u}\left(e^{i t}\right) & =\int_{-\pi}^{\pi} u\left(e^{i \theta}\right) \cdot 2 \operatorname{Re}\left\{\bar{G}_{m}\left(e^{i \theta}, e^{i t}\right)-\frac{1}{4 \pi}\right\} d \theta \\
& =\frac{1}{2 \pi} \cdot \int_{-\pi}^{\pi} u\left(e^{i \theta}\right) \cdot \operatorname{Re}\left\{4 \pi \cdot \bar{G}_{m}\left(e^{i \theta}, e^{i t}\right)-1\right\} d \theta,
\end{aligned}
$$

where $-\pi \leq t \leq \pi$.

(b) Let $f \in L^{2}[-\pi, \pi]$ be a $2 \pi$-periodic real-valued function, with Fourier coefficients $\left\{c_{v}: v= \pm 0, \pm 1, \pm 2, \ldots\right\}$. Since $f(t)=\sum_{v=-\infty}^{\infty} c_{v} \cdot e^{i v t}$ in the $L^{2}$ norm, the function $f(t)$ can be viewed as a function of the unit circle, and therefore the Padé-type approximant $\operatorname{Re}(m /(m+1))_{f}(t)$ to $f(t)$ has the following integral representation:

$$
\begin{aligned}
\operatorname{Re}\left(\frac{m}{m+1}\right)_{f}(t) & =\int_{-\pi}^{\pi} f(\theta) \cdot 2 \operatorname{Re}\left\{\bar{G}_{m}\left(e^{i \theta}, e^{i t}\right)-\frac{1}{4 \pi}\right\} d \theta \\
& =\frac{1}{2 \pi} \cdot \int_{-\pi}^{\pi} f(\theta) \cdot \operatorname{Re}\left\{4 \pi \cdot \bar{G}_{m}\left(e^{i \theta}, e^{i t}\right)-1\right\} d \theta \quad(-\pi \leq t \leq \pi) .
\end{aligned}
$$

Proof. We have

$$
\begin{aligned}
\operatorname{Re}\left(\frac{m}{m+1}\right)_{u}\left(e^{i t}\right)= & 2 \operatorname{Re} T_{u}\left(G_{m}\left(x, e^{i t}\right)\right)-u(0) \\
= & 2 \operatorname{Re} S_{u}\left(\bar{G}_{m}\left(x, e^{i t}\right)\right)-u(0) \\
= & 2 \operatorname{Re} \int_{-\pi}^{\pi} \bar{G}_{m}\left(e^{i \theta}, e^{i t}\right) u\left(e^{i \theta}\right) d \theta-\frac{1}{2 \pi} \int_{-\pi}^{\pi} u\left(e^{i \theta}\right) d \theta \\
= & \frac{1}{2 \pi} \int_{-\pi}^{\pi} u\left(e^{i \theta}\right) \operatorname{Re}\left[4 \pi \bar{G}_{m}\left(e^{i \theta}, e^{i t}\right)\right] d \theta \\
& -\frac{1}{2 \pi} \int_{-\pi}^{\pi} u\left(e^{i \theta}\right) d \theta \\
= & \frac{1}{2 \pi} \cdot \int_{-\pi}^{\pi} u\left(e^{i \theta}\right) \cdot \operatorname{Re}\left\{4 \pi \bar{G}_{m}\left(e^{i \theta}, e^{i t}\right)-1\right\} d \theta \\
= & \int_{-\pi}^{\pi} u\left(e^{i \theta}\right) \cdot 2 \operatorname{Re}\left\{\bar{G}_{m}\left(e^{i \theta}, e^{i t}\right)-\frac{1}{4 \pi}\right\} d \theta \quad(-\pi \leq t \leq \pi) .
\end{aligned}
$$


Setting $z=e^{i t}$ and $\zeta=e^{i \theta}$, we also have

$$
\begin{aligned}
\operatorname{Re}\left(\frac{m}{m+1}\right)_{u}(z) & =\operatorname{Re}\left(\frac{m}{m+1}\right)_{u}\left(e^{i t}\right) \\
& =\operatorname{Re}\left[\frac{1}{2 \pi i} \cdot \int_{-\pi}^{\pi} u\left(e^{i \theta}\right)\left\{\frac{4 \pi \bar{G}_{m}\left(e^{i \theta}, e^{i t}\right)-1}{e^{i \theta}}\right\} i e^{i \theta} d \theta\right] \\
& =\operatorname{Re}\left[\frac{1}{2 \pi i} \cdot \int_{C} u(\zeta)\left\{\frac{4 \pi \bar{G}_{m}(\zeta, z)-1}{\zeta}\right\} d \zeta\right] \\
& =\frac{1}{2 \pi} \cdot \int_{C} u(\zeta) \operatorname{Re}\left\{\frac{4 \pi \bar{G}_{m}(\zeta, z)-1}{i \zeta}\right\} d \zeta \quad(|z|=1)
\end{aligned}
$$

This completes the proof of (a). The proof of (b) is exactly similar and is based on the identification between $L_{\mathbb{R}}^{2}(C)$ and the space of all $2 \pi$ periodic $L^{2}$ real-valued functions on $[-\pi, \pi]$ (every $u(z)=u\left(e^{i t}\right) \in L_{\mathbb{R}}^{2}(C)$ can be interpreted as a $2 \pi$-periodic real-valued function $f(t) \in L^{2}[-\pi, \pi]$ and conversely).

In order to simplify the formalism, we make use of the notation

$$
\begin{aligned}
& \operatorname{Re}\left\{\frac{B_{m}(\zeta, z)}{i \zeta}\right\}:=\operatorname{Re}\left\{\frac{4 \pi \cdot \bar{G}_{m}(\zeta, z)-1}{i \zeta}\right\}, \\
& \operatorname{Re} B_{m}\left(e^{i \theta}, e^{i t}\right):=\operatorname{Re}\left\{4 \pi \cdot \bar{G}_{m}\left(e^{i \theta}, e^{i t}\right)-1\right\} .
\end{aligned}
$$

As it is well known, the function $\operatorname{Re}(m /(m+1))_{u}(z)(|z|=1)$ is continuous (see [6]). Hence, the integral operator $\operatorname{Re}(m /(m+1)) \operatorname{maps} L_{\mathbb{R}}^{2}(C)$ into $L_{\mathbb{R}}^{2}(C)$ and therefore, by the closed graph theorem, it is continuous (of course, under the assumption that $\left|\pi_{m, k}\right|<1$ for all $k \leq m$ ). The integral operator

$$
\begin{gathered}
\operatorname{Re}\left(\frac{m}{m+1}\right): L_{\mathbb{R}}^{2}(C) \longrightarrow L_{\mathbb{R}}^{2}(C) \\
u(z) \longrightarrow \operatorname{Re}\left(\frac{m}{m+1}\right)_{u}(z)=\frac{1}{2 \pi} \cdot \int_{C} u(\zeta) \cdot \operatorname{Re} \frac{B_{m}(\zeta, z)}{i \zeta} d \zeta
\end{gathered}
$$

is called the Padé-type operator for $L_{\mathbb{R}}^{2}(C)$. Its adjoint operator is given by

$$
\begin{gathered}
\operatorname{Re}\left(\frac{m}{m+1}\right)^{*}: L_{\mathbb{R}}^{2}(C) \longrightarrow L_{\mathbb{R}}^{2}(C) \\
u(z) \longrightarrow \operatorname{Re}\left(\frac{m}{m+1}\right)_{u}^{*}(z)=\frac{1}{2 \pi} \cdot \int_{C} u(\zeta) \cdot \operatorname{Re} \frac{B_{m}(z, \zeta)}{i z} d \zeta .
\end{gathered}
$$


In fact, to $\operatorname{Re}(m /(m+1))$ there corresponds a unique operator $\operatorname{Re}(m /(m+$ $1))^{*}: L_{\mathbb{R}}^{2}(C) \rightarrow L_{\mathbb{R}}^{2}(C)$ satisfying $\left\langle\operatorname{Re}(m /(m+1))_{u}, w\right\rangle=\langle u, \operatorname{Re}(m /(m+$ 1) $\left.)_{w}^{*}\right\rangle$, that is,

$$
\int_{C} \operatorname{Re}\left(\frac{m}{m+1}\right)_{u}(\zeta) \cdot w(\zeta) d \zeta=\int_{C} u(z) \cdot \operatorname{Re}\left(\frac{m}{m+1}\right)_{w}^{*}(z) d z
$$

for all $u, w \in L_{\mathbb{R}}^{2}(C)$; since, by Fubini's theorem,

$$
\begin{aligned}
\int_{C} \operatorname{Re}( & \left.\frac{m}{m+1}\right)_{u}(\zeta) \cdot w(\zeta) d \zeta \\
& =\int_{C} \frac{1}{2 \pi} \int_{C} u(z) \cdot \operatorname{Re} \frac{B_{m}(z, \zeta)}{i z} d z w(\zeta) d \zeta \\
& =\int_{C} u(z) \cdot\left(\frac{1}{2 \pi} \int_{C} w(\zeta) \cdot \operatorname{Re} \frac{B_{m}(z, \zeta)}{i z} d \zeta\right) d z
\end{aligned}
$$

we conclude that

$$
\operatorname{Re}\left(\frac{m}{m+1}\right)_{w}^{*}(z)=\frac{1}{2 \pi} \int_{C} w(\zeta) \cdot \operatorname{Re} \frac{B_{m}(z, \zeta)}{i z} d \zeta \quad\left(w \in L_{\mathbb{R}}^{2}(C)\right)
$$

Similarly, as it is pointed out in [6], for any real-valued $2 \pi$-periodic function $f \in L^{2}[-\pi, \pi]$, the Padé-type approximant $\operatorname{Re}(m /(m+1))_{f}(t)$ is continuous, and, by construction, $2 \pi$-periodic. It follows that the integral operator $\operatorname{Re}(m /(m+1))$ maps the space $L_{\mathbb{R},(2 \pi \text {-per })}^{2}[-\pi, \pi]$ of real-valued $2 \pi$-periodic functions of $L^{2}[-\pi, \pi]$ into itself. Hence, by the closed graph theorem, the operator

$$
\begin{gathered}
\operatorname{Re}\left(\frac{m}{m+1}\right): L_{\mathbb{R},(2 \pi \text {-per })}^{2}[-\pi, \pi] \longrightarrow L_{\mathbb{R},(2 \pi \text {-per })}^{2}[-\pi, \pi] \\
f(t) \longrightarrow \operatorname{Re}\left(\frac{m}{m+1}\right)_{f}(t)=\frac{1}{2 \pi} \cdot \int_{-\pi}^{\pi} f(\theta) \cdot \operatorname{Re} B_{m}\left(e^{i \theta}, e^{i t}\right) d \theta
\end{gathered}
$$

is continuous and is called the Padé-type operator for $L_{\mathbb{R},(2 \pi \text {-per })}^{2}[-\pi, \pi]$. Its adjoint operator is then given by

$$
\begin{gathered}
\operatorname{Re}\left(\frac{m}{m+1}\right)^{*}: L_{\mathbb{R},(2 \pi-\text { per })}^{2}[-\pi, \pi] \longrightarrow L_{\mathbb{R},(2 \pi-\text { per })}^{2}[-\pi, \pi] \\
f(t) \longrightarrow \operatorname{Re}\left(\frac{m}{m+1}\right)_{f}^{*}(t)=\frac{1}{2 \pi} \cdot \int_{-\pi}^{\pi} f(\theta) \cdot \operatorname{Re} B_{m}\left(e^{i t}, e^{i \theta}\right) d \theta .
\end{gathered}
$$


In fact, to $\operatorname{Re}(m /(m+1))$ we associate the unique operator $\operatorname{Re}(m /(m+$ $1))^{*}: L_{\mathbb{R},(2 \pi \text {-per })}^{2}[-\pi, \pi] \rightarrow L_{\mathbb{R},(2 \pi \text {-per })}^{2}[-\pi, \pi]$ satisfying

$$
\left\langle\operatorname{Re}\left(\frac{m}{m+1}\right)_{f}, g\right\rangle=\left\langle f, \operatorname{Re}\left(\frac{m}{m+1}\right)_{g}^{*}\right\rangle,
$$

that is,

$$
\int_{-\pi}^{\pi} \operatorname{Re}\left(\frac{m}{m+1}\right)_{f}(t) \cdot g(t) d t=\int_{-\pi}^{\pi} f(\theta) \cdot \operatorname{Re}\left(\frac{m}{m+1}\right)_{g}^{*}(\theta) d \theta
$$

for all $f, g \in L_{\mathbb{R},(2 \pi \text {-per })}^{2}[-\pi, \pi]$; it follows, from Fubini's theorem, that

$$
\begin{aligned}
\int_{-\pi}^{\pi} \operatorname{Re} & \left(\frac{m}{m+1}\right)_{f}(t) \cdot g(t) d t \\
& =\int_{-\pi}^{\pi} \frac{1}{2 \pi} \cdot \int_{-\pi}^{\pi} f(\theta) \cdot \operatorname{Re} B_{m}\left(e^{i \theta}, e^{i t}\right) d \theta g(t) d t \\
& =\int_{-\pi}^{\pi} f(\theta) \cdot\left(\frac{1}{2 \pi} \int_{-\pi}^{\pi} g(t) \cdot \operatorname{Re} B_{m}\left(e^{i \theta}, e^{i t}\right) d t\right) d \theta
\end{aligned}
$$

and consequently

$$
\begin{aligned}
& \operatorname{Re}\left(\frac{m}{m+1}\right)_{g}^{*}(\theta) \\
& \quad=\frac{1}{2 \pi} \cdot \int_{-\pi}^{\pi} g(t) \cdot \operatorname{Re} B_{m}\left(e^{i \theta}, e^{i t}\right) d t \quad\left(g \in L_{\mathbb{R},(2 \pi \text {-per })}^{2}[-\pi, \pi]\right) .
\end{aligned}
$$

Summarizing, we have the following theorem.

THEOREM 2.4. If $m \geq 0$, then for any $u(z) \in L_{\mathbb{R}}^{2}(C)$ and any $f(t) \in$ $L_{\mathbb{R},(2 \pi-\text { per })}^{2}[-\pi, \pi]$, there holds

$$
\begin{aligned}
& \operatorname{Re}\left(\frac{m}{m+1}\right)_{u}^{*}(z)=\frac{1}{2 \pi} \cdot \int_{C} u(\zeta) \cdot \operatorname{Re} \frac{B_{m}(z, \zeta)}{i z} d \zeta, \\
& \operatorname{Re}\left(\frac{m}{m+1}\right)_{f}^{*}(t)=\frac{1}{2 \pi} \cdot \int_{-\pi}^{\pi} f(\theta) \cdot \operatorname{Re} B_{m}\left(e^{i t}, e^{i \theta}\right) d \theta
\end{aligned}
$$

The continuity of the Padé-type operators $\operatorname{Re}(m /(m+1))$ leads immediately to the following convergence results which can be considered as a first example of their application. 
THEOREM 2.5. (a) If the sequence $\left\{u_{n} \in L_{\mathbb{R}}^{2}(C): n=0,1,2, \ldots\right\}$ converges to $u \in L_{\mathbb{R}}^{2}(C)$ in the $L^{2}$-norm, then there holds $\lim _{n \rightarrow \infty} \operatorname{Re}(m /(m+1))_{u_{n}}(z)=$ $\operatorname{Re}(m /(m+1))_{u}(z)$ in the $L^{2}$-norm.

(b) If the sequence $\left\{f_{n} \in L_{\mathbb{R},(2 \pi \text {-per })}^{2}[-\pi, \pi]: n=0,1,2, \ldots\right\}$ converges to $f \in L_{\mathbb{R},(2 \pi \text {-per })}^{2}[-\pi, \pi]$ in the $L^{2}$-norm, then there holds $\lim _{n \rightarrow \infty} \operatorname{Re}(m /(m+$ 1) $)_{f_{n}}(t)=\operatorname{Re}(m /(m+1))_{f}(t)$ in the $L^{2}$-norm.

Corollary 2.6. (a) If the series of functions $u(z)=\sum_{n=0}^{\infty} a_{n} \cdot u_{n}(z)$ (where $\left.a_{n} \in \mathbb{R}, u_{n} \in L_{\mathbb{R}}^{2}(C)\right)$ converges in the $L^{2}$-norm, then $\operatorname{Re}(m /(m+1))_{u}(z)=$ $\sum_{n=0}^{\infty} a_{n} \operatorname{Re}(m /(m+1)) u_{n}(z)$ in the $L^{2}$-norm.

(b) If the series of functions $f(t)=\sum_{n=0}^{\infty} a_{n} \cdot f_{n}(t)$ (where $a_{n} \in \mathbb{R}, f_{n} \in$ $\left.L_{\mathbb{R},(2 \pi \text {-per })}^{2}[-\pi, \pi]\right)$ converges in the $L^{2}$-norm then $\operatorname{Re}(m /(m+1))_{f}(t)=$ $\sum_{n=0}^{\infty} a_{n} \operatorname{Re}(m /(m+1))_{f_{n}}(t)$ in the $L^{2}$-norm.

Now we determine the conditions under which the integral operator $\operatorname{Re}(m /(m+1))$ is compact onto $L_{\mathbb{R},(2 \pi \text {-per })}^{2}[-\pi, \pi]$. Since, for each fixed $t \in[-\pi, \pi]$, the kernel function $\operatorname{Re} B_{m}\left(e^{i \theta}, e^{i t}\right)$ is bounded in $\theta$, it follows, from Tonelli's theorem, that the following theorem holds true.

THEOREM 2.7. If there is a constant $c_{*}<\infty$ such that

$$
\int_{-\pi}^{\pi}\left|\operatorname{Re} B_{m}\left(e^{i \theta}, e^{i t}\right)\right|^{2} d \theta \leq(2 \pi)^{2} \cdot c_{*}
$$

for almost all $t \in[-\pi, \pi]$, then the Padé-type operator $\operatorname{Re}(m /(m+1))$ : $L_{\mathbb{R},(2 \pi \text {-per })}^{2}[-\pi, \pi] \rightarrow L_{\mathbb{R},(2 \pi \text {-per })}^{2}[-\pi, \pi]$ is compact. Moreover,

$$
\left\|\operatorname{Re}\left(\frac{m}{m+1}\right)\right\| \leq(2 \pi)^{5 / 2} \cdot c_{*}
$$

and $\operatorname{Re}(m /(m+1))^{*}$ is also compact.

It is readily seen that if the Padé-type operator $\operatorname{Re}(m /(m+1))$ : $L_{\mathbb{R},(2 \pi \text {-per })}^{2}[-\pi, \pi] \rightarrow L_{\mathbb{R},(2 \pi \text {-per })}^{2}[-\pi, \pi]$ is compact, then it is not one-toone. This follows from the fact that $\operatorname{dim} L_{\mathbb{R},(2 \pi \text {-per })}^{2}[-\pi, \pi]=\infty$ and therefore 0 must be an eigenvalue of $\operatorname{Re}(m /(m+1))$. However, it would be interesting to know a necessary and sufficient condition under which for any $h \in L_{\mathbb{R},(2 \pi \text {-per })}^{2}[-\pi, \pi]$ there is an $f \in L_{\mathbb{R},(2 \pi \text {-per })}^{2}[-\pi, \pi]$ such that $\operatorname{Re}(m /(m+1))_{f}=h$. Of course, such a general condition is the inequality 


$$
\left\|\operatorname{Re}\left(\frac{m}{m+1}\right)_{f}^{*}\right\|_{2} \geq c \cdot\|f\|_{2}
$$

or alternatively,

$$
\int_{-\pi}^{\pi}|f(t)|^{2} d t \leq c \cdot \int_{-\pi}^{\pi}\left|\int_{-\pi}^{\pi} f(\theta) \cdot \operatorname{Re} B_{m}\left(e^{i t}, e^{i \theta}\right) d \theta\right|^{2} d t
$$

for some constant $c>0$ and for every $f \in L_{\mathbb{R},(2 \pi \text {-per })}^{2}[-\pi, \pi]$. Obviously, this inequality is true if and only if

$$
|f(t)| \leq c \cdot\left|\int_{-\pi}^{\pi} f(\theta) \cdot \operatorname{Re} B_{m}\left(e^{i t}, e^{i \theta}\right) d \theta\right|
$$

for almost all $t \in[-\pi, \pi]$, and thus we have proved the following theorem describing a sufficient condition under which every function in $L_{\mathbb{R},(2 \pi \text {-per })}^{2}[-\pi, \pi]$ is a Padé-type approximant.

THEOREM 2.8. If there is a constant $c>0$ such that

$$
|f(t)| \leq c \cdot\left|\int_{-\pi}^{\pi} f(\theta) \cdot \operatorname{Re} B_{m}\left(e^{i t}, e^{i \theta}\right) d \theta\right|
$$

almost everywhere on $[-\pi, \pi]$, for every $f \in L_{\mathbb{R},(2 \pi \text {-per })}^{2}[-\pi, \pi]$, then the range of $\operatorname{Re}(m /(m+1))$ equals $L_{\mathbb{R},(2 \pi \text {-per })}^{2}[-\pi, \pi]$.

Finally, we turn to integral representation formulas in the harmonic case. If $u$ is harmonic and real-valued in the unit disk, then, for any $0 \leq r<1$, the restriction $u_{r}(t)=u\left(r \cdot e^{i t}\right)(-\pi \leq t \leq \pi)$ of $u(z)$ to the circle of radius $r$ can be interpreted as a real-valued, $2 \pi$-periodic function in $L^{2}[-\pi, \pi]$. According to Theorem 2.3, the Padé-type approximant $\operatorname{Re}(m /(m+1))_{u_{r}}(t)$ to $u_{r}(t)$ is given by the integral representation formula

$$
\begin{aligned}
\operatorname{Re}\left(\frac{m}{m+1}\right)_{u_{r}}(t) & =\frac{1}{2 \pi} \cdot \int_{-\pi}^{\pi} u_{r}(\theta) \cdot \operatorname{Re}\left\{4 \pi \cdot \bar{G}_{m}\left(r \cdot e^{i \theta}, r \cdot e^{i t}\right)-1\right\} d \theta \\
& =\frac{1}{2 \pi} \cdot \int_{-\pi}^{\pi} u_{r}\left(r \cdot e^{i \theta}\right) \cdot \operatorname{Re}\left\{4 \pi \cdot \bar{G}_{m}\left(r \cdot e^{i \theta}, r \cdot e^{i t}\right)-1\right\} d \theta .
\end{aligned}
$$


62 Integral representations for Padé-type operators

After a simple change of variables $z=r \cdot e^{i t}$ and $\zeta=r \cdot e^{i \theta}$, we obtain

$$
\begin{aligned}
\operatorname{Re}\left(\frac{m}{m+1}\right)_{u}(z) & =\frac{1}{2 \pi} \cdot \int_{|\zeta|=r} u(\zeta) \cdot \operatorname{Re}\left\{\frac{4 \pi \cdot \bar{G}_{m}(\zeta, z)-1}{\zeta i}\right\} d \zeta \\
& =\frac{1}{2 i} \cdot \int_{|\zeta|=r} u(\zeta) \cdot \operatorname{Re}\left\{\frac{B_{m}(\zeta, z)}{\zeta i}\right\} d \zeta,
\end{aligned}
$$

and hence we can state the following theorem.

THEOREM 2.9. Let $M=\left(\pi_{m, k}\right)_{m \geq 0,0 \leq k \leq m}$ be an infinite triangular interpolation matrix with complex entries and, for any $m \geq 0$, let $G_{m}(x, z)$ be the unique polynomial of degree at most $m$ which interpolates the function $(1-x \cdot z)^{-1}$ at $x=$ $\pi_{m, 0}, \pi_{m, 1}, \pi_{m, 2}, \ldots, \pi_{m, m}$ (where $z$ is fixed and $\left|\pi_{m, k}\right|<1$ for each $k \leq m)$.

The Padé-type approximant $\operatorname{Re}(m /(m+1))_{u}(z)$ to the harmonic real-valued function $u(z)$ in the disk is given by the following integral representation formula:

$$
\operatorname{Re}\left(\frac{m}{m+1}\right)_{u}(z)=\frac{1}{2 \pi} \cdot \int_{|\zeta|=|z|} u(\zeta) \cdot \operatorname{Re}\left\{\frac{B_{m}(\zeta, z)}{i \zeta}\right\} d \zeta \quad(z \in D)
$$

As it is mentioned in [5], the function $\operatorname{Re}(m /(m+1))_{u}(z)$ is the real part of an analytic function in the unit disk, and therefore, it is a harmonic real-valued function in $D$ (of course, under the assumption that $\left|\pi_{m, k}\right|<1$ for all $\left.k \leq m\right)$. If $H_{\mathbb{R}}(D)$ is the space of all harmonic real-valued functions in $D$, the integral operator

$$
\begin{gathered}
\operatorname{Re}\left(\frac{m}{m+1}\right): H_{\mathbb{R}}(D) \longrightarrow H_{\mathbb{R}}(D) \\
u(z) \longrightarrow \operatorname{Re}\left(\frac{m}{m+1}\right)_{u}(z)=\frac{1}{2 \pi} \cdot \int_{|\zeta|=|z|} u(\zeta) \cdot \operatorname{Re}\left\{\frac{B_{m}(\zeta, z)}{i \zeta}\right\} d \zeta
\end{gathered}
$$

is said to be a Padé-type operator of $H_{\mathbb{R}}(D)$. It is easily seen that a Padétype operator of $H_{\mathbb{R}}(D)$ is continuous. For, if $\left\{u_{n} \in H_{\mathbb{R}}(D): n=0,1,2, \ldots\right\}$ and if $\lim _{n \rightarrow \infty} u_{n}=u \in H_{\mathbb{R}}(D)$ compactly in the disk $D$, then, by the 
maximum principle for harmonic functions, we have

$$
\begin{aligned}
& \sup _{|z| \leq r} \mid \operatorname{Re}\left(\frac{m}{m+1}\right)_{u_{n}}(z)-\operatorname{Re}\left(\frac{m}{m+1}\right)_{u}(z) \mid \\
&=\sup _{|z|=r}\left|\operatorname{Re}\left(\frac{m}{m+1}\right)_{u_{n}}(z)-\operatorname{Re}\left(\frac{m}{m+1}\right)_{u}(z)\right| \\
&=\frac{1}{2 \pi} \cdot \sup _{|z|=r}\left|\int_{|\zeta|=r}\left[u_{n}(\zeta)-u(\zeta)\right] \cdot \operatorname{Re}\left\{\frac{B_{m}(\zeta, z)}{\zeta i}\right\} d \zeta\right| \\
& \leq \frac{1}{2 \pi r} \cdot 2 \pi r \cdot\left\{\sup _{|z|=r,|\zeta|=r}\left|\operatorname{Re} B_{m}(\zeta, z)\right|\right\} \cdot\left\{\sup _{|\zeta|=r}\left|u_{n}(\zeta)-u(\zeta)\right|\right\} \\
& \leq L(r, m) \cdot\left\{\sup _{|\zeta|=r}\left|u_{n}(\zeta)-u(\zeta)\right|\right\}
\end{aligned}
$$

for any $r<1$, and the continuity of $\operatorname{Re}(m /(m+1)): H_{\mathbb{R}}(D) \rightarrow H_{\mathbb{R}}(D)$ follows.

As for the $L^{2}$-case, the continuity of the Padé-type operator for $H_{\mathbb{R}}(D)$ leads to the following convergence results.

THEOREM 2.10. If the sequence $\left\{u_{n}: n=0,1,2, \ldots\right\}$ of harmonic real-valued functions in the open unit disk converges compactly to $u \in H_{\mathbb{R}}(D)$, then there holds

$$
\lim _{n \rightarrow \infty} \operatorname{Re}\left(\frac{m}{m+1}\right)_{u_{n}}(z)=\operatorname{Re}\left(\frac{m}{m+1}\right)_{u}(z)
$$

compactly in D.

COROLlary 2.11. If the series of harmonic real-valued functions

$$
u(z)=\sum_{n=0}^{\infty} a_{n} \cdot u_{n}(z) \quad\left(a_{n} \in \mathbb{R}, u_{n} \in H_{\mathbb{R}}(D)\right)
$$

converges compactly in the disk, then

$$
\operatorname{Re}\left(\frac{m}{m+1}\right)_{u}(z)=\sum_{n=0}^{\infty} a_{n} \operatorname{Re}\left(\frac{m}{m+1}\right)_{u_{n}}(z)
$$

the convergence of the series being compact in $D$. 
64 Integral representations for Padé-type operators

Remark 2.12. In [2], Brezinski showed that the (Hermite) interpolation polynomial $G_{m}(x, z)$ of $(1-x z)^{-1}$ at $x=\pi_{m, 0}, \pi_{m, 1}, \ldots, \pi_{m, m}$ is given by

$$
G_{m}(x, z)=\frac{1}{1-x \cdot z} \cdot\left(1-\frac{v_{m+1}(x)}{v_{m+1}\left(z^{-1}\right)}\right) \quad\left(z \neq \pi_{m, k}^{-1}, k=0,1, \ldots, m\right),
$$

where $v_{m+1}(x)$ is any generating polynomial $v_{m+1}(x)=\gamma \cdot \prod_{k=0}^{m}\left(x-\pi_{m, k}\right)$ $(\gamma \neq 0)$. We thus obtain the following expressions for the kernels $\operatorname{Re}\left\{B_{m}(\zeta, z) / \zeta i\right\}$ and $\operatorname{Re} B_{m}\left(e^{i \theta}, e^{i t}\right)$ :

$$
\begin{aligned}
& \operatorname{Re}\left\{\frac{B_{m}(\zeta, z)}{\zeta i}\right\}=\operatorname{Re}\left\{\frac{-4 i \zeta^{-1}}{1-\zeta \cdot \bar{z}}\left(1-\bar{z}^{m+1} \cdot \prod_{k=0}^{m} \frac{\zeta-\overline{\pi_{m, k}}}{1-\overline{z \cdot \pi_{m, k}}}\right)-\zeta^{-1}\right\}, \\
& \operatorname{Re} B_{m}\left(e^{i \theta}, e^{i t}\right)=\operatorname{Re}\left\{\frac{4 \pi}{1-e^{i(\theta-t)}}\left(1-\prod_{k=0}^{m} \frac{e^{i \theta}-\overline{\pi_{m, k}}}{e^{i t}-\overline{\pi_{m, k}}}\right)-1\right\} .
\end{aligned}
$$

If, for example, $\pi_{m, 0}=\cdots=\pi_{m, m}=0$, then for any $u \in L_{\mathbb{R}}^{2}(C)$, we have

$$
\begin{aligned}
& \operatorname{Re}\left(\frac{m}{m+1}\right)_{u}(z) \\
& \quad=\operatorname{Re}\left\{\frac{2}{\pi i} \cdot \sum_{v=0}^{m} \bar{z}^{v} \int_{C} u(\zeta) \cdot \zeta^{v-1} d \zeta-\frac{2}{\pi i} \cdot \int_{C} u(\zeta) \cdot \zeta^{-1} d \zeta\right\} \quad(z \in C)
\end{aligned}
$$

or

$$
\begin{aligned}
& \operatorname{Re}\left(\frac{m}{m+1}\right)_{u}\left(e^{i t}\right) \\
& =2 \cdot \int_{-\pi}^{\pi} u\left(e^{i \theta}\right) \cos (\theta-t) d \theta-2 \cdot \int_{-\pi}^{\pi} u\left(e^{i \theta}\right) \cos [m-(\theta-t)] d \theta \\
& =4 \cdot \int_{-\pi}^{\pi} u\left(e^{i \theta}\right) \cdot \sin \left[\frac{(m+1) \theta-(m+1) t}{2}\right] \\
& \cdot \sin \left[\frac{(m-1) \theta-(m-1) t}{2}\right] d \theta \quad(-\pi \leq t \leq \pi) .
\end{aligned}
$$

\section{Integral representations and composed Padé-type approximation}

We are now in a position to generalize the definitions and results of Section 2 to the context of composed Padé-type approximation. Recall that a composed Padé-type approximant to a harmonic complex-valued function $u=u_{1}+i u_{2}$ in the disk $D$ (resp., to an $L^{p}$ complex-valued 
function $u=u_{1}+i u_{2}$ on the circle $C$ or to a $2 \pi$-periodic complex-valued function $\left.f=f_{1}+i f_{2} \in L^{p}[-\pi, \pi]\right)$ is a coordinate approximant given by the formula

$$
\left(\frac{m}{m+1}\right)_{u}(z)=\operatorname{Re}\left(\frac{m_{1}}{m_{1}+1}\right)_{u_{1}}(z)+i \operatorname{Re}\left(\frac{m_{2}}{m_{2}+1}\right)_{u_{2}}(z) \quad(z \in D),
$$

respectively, by the formula

$$
\left(\frac{m}{m+1}\right)_{u}(z)=\operatorname{Re}\left(\frac{m_{1}}{m_{1}+1}\right)_{u_{1}}(z)+i \operatorname{Re}\left(\frac{m_{2}}{m_{2}+1}\right)_{u_{2}}(z) \quad(z \in C)
$$

or

$$
\left(\frac{m}{m+1}\right)_{f}(t)=\operatorname{Re}\left(\frac{m_{1}}{m_{1}+1}\right)_{f_{1}}(z)+i \operatorname{Re}\left(\frac{m_{2}}{m_{2}+1}\right)_{f_{2}}(t)
$$

where $-\pi \leq t \leq \pi$ (see [7]).

Set

$$
\begin{aligned}
L_{\mathbb{C}}^{p}(C):= & \left\{u \in L^{p}(C): u \text { is complex-valued }\right\}, \\
L_{\mathbb{C},(2 \pi \text {-per })}^{p}[-\pi, \pi]:= & \left\{f \in L^{p}[-\pi, \pi]: f\right. \text { is complex-valued and } \\
& 2 \pi \text {-periodic }(f(-\pi)=f(\pi))\}, \\
H_{\mathbb{C}}(D):= & \{u: D \longrightarrow \mathbb{C}: u \text { is harmonic and complex-valued }\} .
\end{aligned}
$$

From Theorems 2.3 and 2.9, the following theorem follows immediately.

THEOREM 3.1. For $j=1,2$, let $M^{(j)}=\left(\pi_{m_{i}, k}^{(j)}\right)_{m_{j} \geq 0,0 \leq k \leq m_{j}}$ be an infinite triangular interpolation matrix with complex entries $\pi_{m_{j}, k}^{(j)} \in D$, and, for any $m_{j} \geq 0$, let $G_{m_{j}}^{(j)}(x, z)$ be the unique polynomial of degree at most $m_{j}$ which interpolates the function $(1-x z)^{-1}$ at $x=\pi_{m_{j}, 0}^{(j)}, \pi_{m_{j}, 1}^{(j)}, \ldots, \pi_{m_{j}, m_{j}}^{(j)}$ (where $z$ is regarded as a parameter).

$$
\begin{gathered}
\text { If } G_{m_{j}}^{(j)}(x, z)=\sum_{v=0}^{m_{j}} g_{v}^{\left(j, m_{j}\right)}(z) \cdot x^{v} \text {, denote by } \overline{G_{m_{j}}^{(j)}}(x, z) \text { the polynomial } \\
\sum_{v=0}^{m_{j}} \overline{g_{v}^{\left(j, m_{j}\right)}(z)} \cdot x^{v} .
\end{gathered}
$$

Put

$$
B_{m_{j}}^{(j)}(x, z)=4 \pi \cdot \overline{G_{m_{j}}^{(j)}}(x, z)-1
$$


66 Integral representations for Padé-type operators

(a) For any $u=u_{1}+i \cdot u_{2} \in L_{\mathbb{C}}^{2}(C)$, the corresponding composed Padé-type approximant $(m /(m+1))_{u}(z)$ to $u(z)$ has the following integral representation

$$
\begin{aligned}
\left(\frac{m}{m+1}\right)_{u}(z)=\frac{1}{2 \pi} \cdot \int_{C}\{ & u_{1}(\zeta) \cdot \operatorname{Re}\left[\frac{B_{m_{1}}^{(1)}(\zeta, z)}{\zeta i}\right] \\
& \left.+i \cdot u_{2}(z) \cdot \operatorname{Re}\left[\frac{B_{m_{2}}^{(2)}(\zeta, z)}{i \zeta}\right]\right\} d \zeta \quad(|z|=1),
\end{aligned}
$$

or equivalently

$$
\begin{aligned}
\left(\frac{m}{m+1}\right)_{u}\left(e^{i t}\right)=\frac{1}{2 \pi} \cdot \int_{-\pi}^{\pi}\{ & u_{1}\left(e^{i \theta}\right) \cdot \operatorname{Re} B_{m_{1}}^{(1)}\left(e^{i \theta}, e^{i t}\right) \\
& \left.+i \cdot u_{2}\left(e^{i \theta}\right) \cdot \operatorname{Re} B_{m_{2}}^{(2)}\left(e^{i \theta}, e^{i t}\right)\right\} d \theta \quad(-\pi \leq t \leq \pi) .
\end{aligned}
$$

(b) For any $f=f_{1}+i \cdot f_{2} \in L_{\mathbb{C},(2 \pi \text {-per })}^{2}[-\pi, \pi]$, the corresponding composed Padé-type approximant $(m /(m+1))_{f}(t)$ to $f(t)$ has the following integral representation:

$$
\begin{aligned}
\left(\frac{m}{m+1}\right)_{f}(t)=\frac{1}{2 \pi} \cdot \int_{-\pi}^{\pi}\{ & f_{1}(\theta) \cdot \operatorname{Re} B_{m_{1}}^{(1)}\left(e^{i \theta}, e^{i t}\right) \\
& \left.+i \cdot f_{2}(\theta) \cdot \operatorname{Re} B_{m_{2}}^{(2)}\left(e^{i \theta}, e^{i t}\right)\right\} d \theta \quad(-\pi \leq t \leq \pi) .
\end{aligned}
$$

(c) For any $u=u_{1}+i \cdot u_{2} \in H_{\mathbb{C}}(D)$, the corresponding composed Padé-type approximant $(m /(m+1))_{u}(z)$ to $u(z)$ has the following integral representation:

$$
\begin{aligned}
\left(\frac{m}{m+1}\right)_{u}(z)=\frac{1}{2 \pi} \cdot \int_{|\zeta|=|z|}\{ & u_{1}(z) \cdot \operatorname{Re}\left[\frac{B_{m_{1}}^{(1)}(\zeta, z)}{\zeta i}\right] \\
& \left.+i \cdot u_{2}(\zeta) \cdot \operatorname{Re}\left[\frac{B_{m_{2}}^{(2)}(\zeta, z)}{\zeta i}\right]\right\} d \zeta \quad(|z|<1) .
\end{aligned}
$$

In particular, since any Padé-type approximant in the ordinary sense is a composed Padé-type approximant, we can give integral representation for the classical Padé-type approximants to analytic functions.

Corollary 3.2. Let $M=\left(\pi_{m, k}\right)_{m \geq 0,0 \leq k \leq m}$ be an infinite triangular interpolation matrix with complex entries $\pi_{m, k} \in D$, and, for any $m \geq 0$, let $G_{m}(x, z)$ 
be the unique polynomial of degree at most $m$ which interpolates the function $(1-x z)^{-1}$ at $x=\pi_{m, 0}, \pi_{m, 1}, \ldots, \pi_{m, m}$ ( $z$ is regarded as a parameter $)$.

If $G_{m}(x, z)=\sum_{v=0}^{m} g_{v}^{(m)}(z) \cdot x^{v}$, denote by $\bar{G}_{m}(x, z)$ the polynomial $\sum_{v=0}^{m} \overline{g_{v}^{(m)}(z)} \cdot x^{v}$, and put

$$
B_{m}(x, z)=4 \pi \cdot \bar{G}_{m}(x, z)-1 .
$$

For any $f \in O(D)$, the corresponding Padé-type approximant $(m /(m+$ $1))_{f}(z)$ to $f(z)$ (in the Brezinski's sense of [1]) has the following integral representation:

$$
\left(\frac{m}{m+1}\right)_{f}(z)=\frac{1}{2 \pi} \cdot \int_{|\zeta|=|z|} f(\zeta) \cdot \operatorname{Re}\left[\frac{B_{m}(\zeta, z)}{\zeta i}\right] d \zeta \quad(|z|<1) .
$$

Under the assumptions of Theorem 3.1, the integral operators

$$
\begin{aligned}
\left(\frac{m}{m+1}\right) & : L_{\mathbb{C}}^{2}(C) \longrightarrow L_{\mathbb{C}}^{2}(C) \\
u & =u_{1}+i u_{2} \longmapsto\left(\frac{m}{m+1}\right)_{u}(z) \\
& =\frac{1}{2 i} \cdot \int_{C}\left\{u_{1}(\zeta) \cdot \operatorname{Re}\left[\frac{B_{m_{1}}^{(1)}(\zeta, z)}{\zeta i}\right]+i \cdot u_{2}(\zeta) \cdot \operatorname{Re}\left[\frac{B_{m_{2}}^{(2)}(\zeta, z)}{\zeta i}\right]\right\} d \zeta, \\
\left(\frac{m}{m+1}\right) & : L_{\mathbb{C},(2 \pi-\text { per })}^{2}[-\pi, \pi] \longrightarrow L_{\mathbb{C},(2 \pi-\text { per })}^{2}[-\pi, \pi] ; \\
f & =f_{1}+i \cdot f_{2} \longmapsto\left(\frac{m}{m+1}\right)_{f}(t) \\
& =\frac{1}{2 \pi} \cdot \int_{-\pi}\left\{f_{1}(\theta) \cdot \operatorname{Re} B_{m_{1}}^{(1)}\left(e^{i \theta}, e^{i t}\right)+i \cdot f_{2}(\theta) \cdot \operatorname{Re} B_{m_{2}}^{(2)}\left(e^{i \theta}, e^{i t}\right)\right\} d \theta, \\
\left(\frac{m}{m+1}\right): & H_{\mathbb{C}}(D) \longrightarrow H_{\mathbb{C}}(D) ; \\
u & =u_{1}+i u_{2} \longmapsto\left(\frac{m}{m+1}\right)_{u}(z) \\
& =\frac{1}{2 \pi} \cdot \int_{|\zeta|=|z|}\left\{u_{1}(\zeta) \cdot \operatorname{Re}\left[\frac{B_{m_{1}}^{(1)}(\zeta, z)}{\zeta i}\right]\right. \\
& \left.+i \cdot u_{2}(\zeta) \cdot \operatorname{Re}\left[\frac{B_{m_{2}}^{(2)}(\zeta, z)}{\zeta i}\right]\right\} d \zeta
\end{aligned}
$$


are called composed Padé-type operators for $L_{\mathbb{C}}^{2}, L_{\mathbb{C},(2 \pi \text {-per })}^{2}[-\pi, \pi]$, and $H_{\mathbb{C}}(D)$, respectively. Under the assumptions of Corollary 3.2, the integral operator

$$
\begin{gathered}
\left(\frac{m}{m+1}\right): O(D) \longrightarrow O(D) \\
f \longmapsto\left(\frac{m}{m+1}\right)_{f}(z)=\frac{1}{2 \pi} \cdot \int_{|\zeta|=|z|} f(\zeta) \cdot \operatorname{Re}\left[\frac{B_{m}(\zeta, z)}{\zeta i}\right] d \zeta
\end{gathered}
$$

is called a Padé-type operator for $O(D)$.

The continuity of these integral operators follows directly from the arguments of Section 2 and leads to the following result.

THEOREM 3.3. Under the assumptions and notations of Theorem 3.1 and Corollary 3.2,

(a) if the sequence $\left\{u_{n} \in L_{\mathbb{C}}^{2}(C): n=0,1,2, \ldots\right\}$ converges to $u \in L_{\mathbb{C}}^{2}(C)$ in the $L^{2}$-norm, then $\lim _{n \rightarrow \infty}(m /(m+1))_{u_{n}}(z)=(m /(m+1))_{u}(z)$ in the $L^{2}$-norm;

(b) if the sequence $\left\{f_{n} \in L_{\mathbb{C},(2 \pi \text {-per })}^{2}[-\pi, \pi]: n=0,1,2, \ldots\right\}$ converges to $f \in L_{\mathbb{C},(2 \pi \text {-per })}^{2}[-\pi, \pi]$, with respect to the $L^{2}$-norm, then $\lim _{n \rightarrow \infty}(m)$ $(m+1))_{f_{n}}(t)=(m /(m+1))_{f}(t)$ in the $L^{2}$-norm;

(c) if the sequence $\left\{u_{n} \in H_{\mathbb{C}}(D): n=0,1,2, \ldots\right\}$ converges to $u \in H_{\mathbb{C}}(D)$ compactly in $D$, then $\lim _{n \rightarrow \infty}(m /(m+1))_{u_{n}}(z)=(m /(m+1))_{u}(z)$ compactly in $D$;

(d) if the sequence $\left\{f_{n} \in O(D): n=0,1,2, \ldots\right\}$ converges to $f \in O(D)$ compactly in $D$, then $\lim _{n \rightarrow \infty}(m /(m+1))_{f_{n}}(z)=(m /(m+1))_{f}(z)$ compactly in $D$.

Especially, for series of functions, there is an obvious consequence of this theorem.

Corollary 3.4. Under the assumptions of Theorem 3.1 and Corollary 3.2,

(a) if the series of functions $u(z)=\sum_{n=0}^{\infty} a_{n} \cdot u_{n}(z)\left(a_{n} \in \mathbb{C}, u_{n} \in L_{\mathbb{C}}^{2}(C)\right)$ converges in the $L^{2}$-norm, then $(m /(m+1))_{u}(z)=\sum_{n=0}^{\infty} a_{n} \cdot(m /(m+$ 1) $)_{u_{n}}(z)$ in the $L^{2}$-norm;

(b) if the series of functions $f(t)=\sum_{n=0}^{\infty} a_{n} \cdot f_{n}(t)$ (where $a_{n} \in \mathbb{C}, f_{n} \in$ $\left.L_{\mathbb{C},(2 \pi \text {-per })}^{2}[-\pi, \pi]\right)$ converges in the $L^{2}$-norm, then $(m /(m+$ 1) $)_{f}(t) \sum_{n=0}^{\infty} a_{n} \cdot(m /(m+1))_{f_{n}}(t)$ in the $L^{2}$-norm;

(c) if the series of functions $u(z)=\sum_{n=0}^{\infty} a_{n} \cdot u_{n}(z)\left(a_{n} \in \mathbb{C}, u_{n} \in H_{\mathbb{C}}(D)\right)$ converges compactly in the disk $D$, then $(m /(m+1))_{u}(z)=\sum_{n=0}^{\infty} a_{n}$. $(m /(m+1))_{u_{n}}(z)$ compactly in $D$; 
(d) if the series of analytic functions $f(z)=\sum_{n=0}^{\infty} a_{n} \cdot f_{n}(z)\left(a_{n} \in \mathbb{C}\right.$, $\left.f_{n} O(D)\right)$ converges compactly in $D$, then $(m /(m+1))_{f}(z)=\sum_{n=0}^{\infty} a_{n}$. $(m /(m+1))_{f_{n}}(z)$ compactly in $D$.

Remark 3.5. Padé and Padé-type approximants to arbitrary series of functions were first considered by Brezinski in $[1,2]$.

\section{References}

[1] C. Brezinski, Rational approximation to formal power series, J. Approx. Theory 25 (1979), no. 4, 295-317.

[2] _ Padé approximants: old and new, Yearbook: Surveys of Mathematics 1983, Bibliographisches Institut, Mannheim, 1983, pp. 37-63.

[3] , Duality in Padé-type approximation, J. Comput. Appl. Math. 30 (1990), no. 3, 351-357.

[4] N. J. Daras, Continuity of distributions and global convergence of Padé-type approximants in Runge domains, Indian J. Pure Appl. Math. 26 (1995), no. 2, 121-130.

[5]_ Rational approximation to harmonic functions, Numer. Algorithms 20 (1999), no. 4, 285-301.

[6] , Padé and Padé-type approximation for $2 \pi$-periodic $L^{p}$ functions, Acta Appl. Math. 62 (2000), no. 3, 245-343.

[7] _ Composed Padé-type approximation, J. Comput. Appl. Math. 134 (2001), no. 1-2, 95-112.

Nicholas J. Daras: Department of Mathematics, Hellenic Air Force Academy, Dekeleia Attikis, Greece

Current address: Jean Moreas 19, 15232 Chalandri, Athens, Greece

E-mail address: njdaras@myflash.gr 


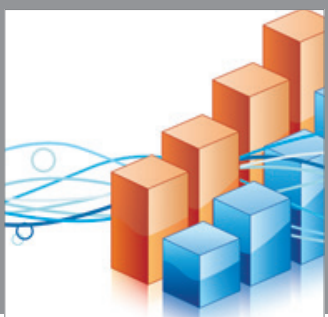

Advances in

Operations Research

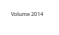

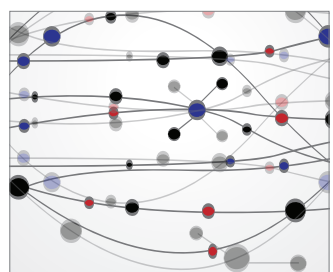

\section{The Scientific} World Journal
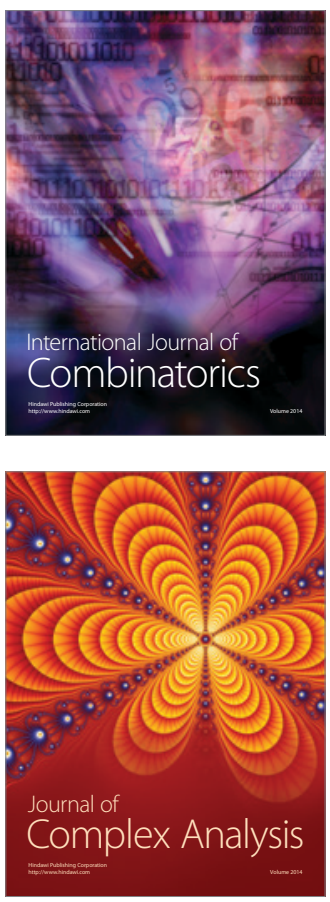

International Journal of

Mathematics and

Mathematical

Sciences
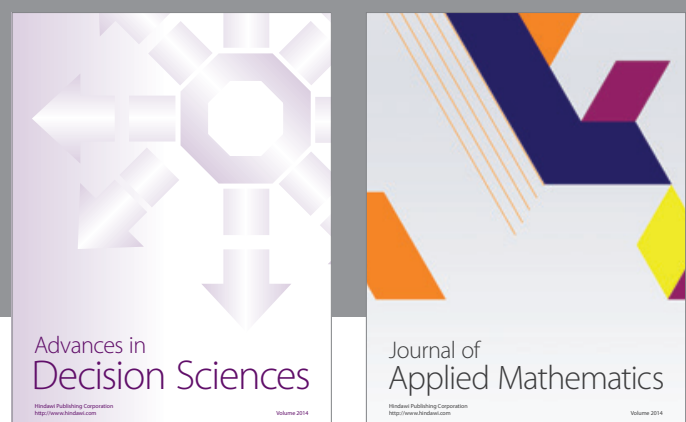

Journal of

Applied Mathematics
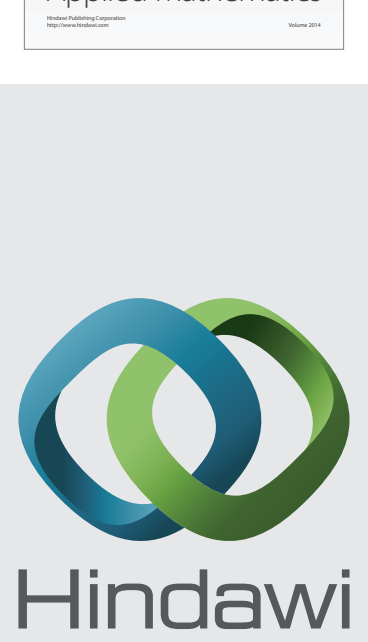

Submit your manuscripts at http://www.hindawi.com
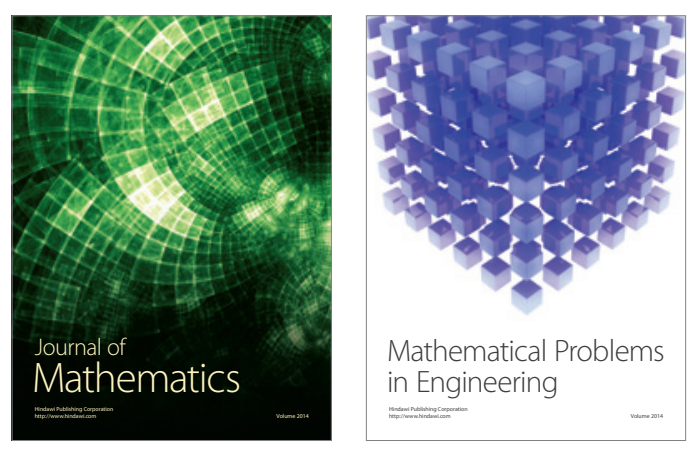

Mathematical Problems in Engineering
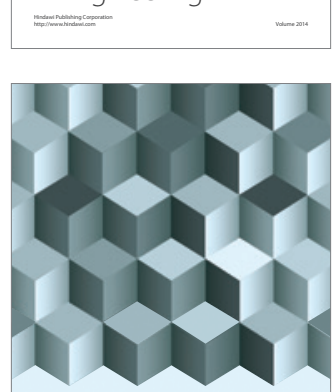

Journal of

Function Spaces
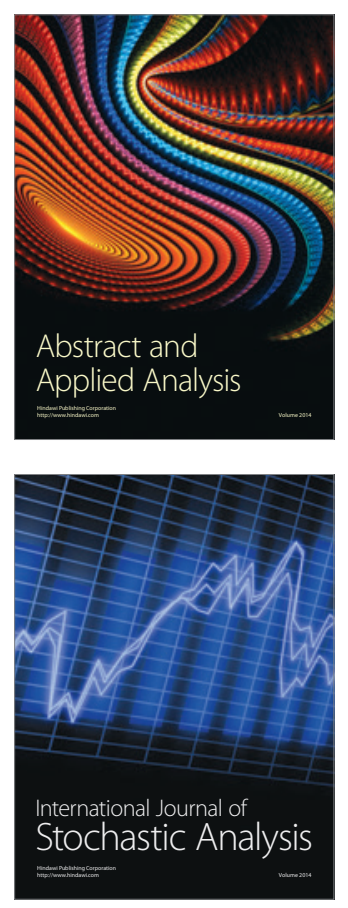

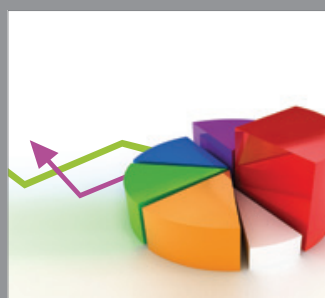

ournal of

Probability and Statistics

Promensencen
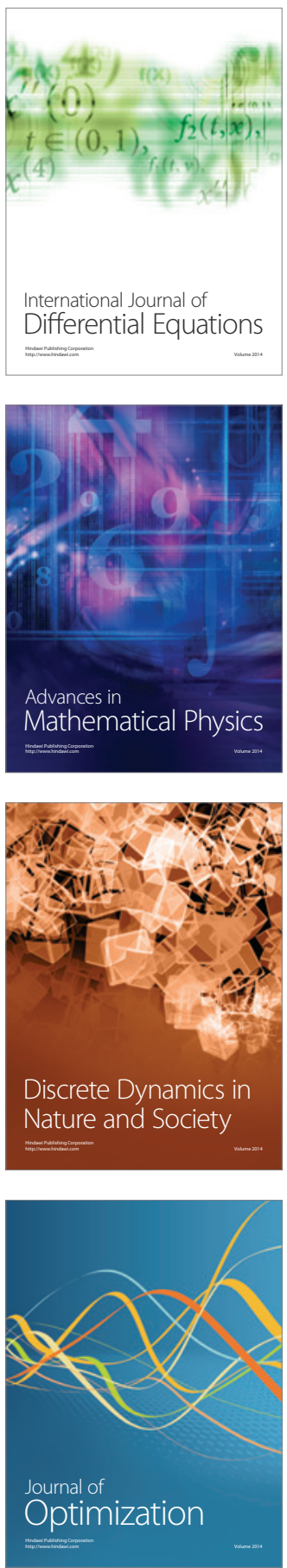